\title{
Colorimetric characteristics of the phenolic fractions obtained from Tempranillo and Graciano wines through the use of different instrumental techniques
}

\author{
Matilde García-Marino a ${ }^{\text {, M. Luisa Escudero-Gilete }}{ }^{\mathrm{b}}$, M. Teresa Escribano-Bailón ${ }^{\mathrm{a}}$, \\ M. Lourdes González-Miret ${ }^{\mathrm{b}}$, Julián C. Rivas-Gonzalo ${ }^{\mathrm{a}}$, Francisco J. Heredia ${ }^{\mathrm{b}, *}$

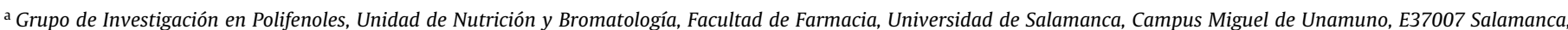 \\ Spain \\ ${ }^{\mathrm{b}}$ Food Colour and Quality Lab., Dept. Nutrition and Food Science. Universidad de Sevilla. Facultad de Farmacia, E41012 Sevilla, Spain
}

\section{A R T I C L E I N F O}

\section{Article history:}

Received 18 January 2012

Received in revised form 7 March 2012

Accepted 13 March 2012

Available online 29 March 2012

\section{Keywords:}

Wine colour

Anthocyanins

Tempranillo

Graciano

Colorimetric techniques

CIELAB

\begin{abstract}
A B S T R A C T
The aim of the present work was to determine whether there is any relationship between measurement by transmission and reflection (in the latter case, with and without contact with the sample). We also evaluated which methodology used would offer a better interpretation of the results in visual terms. For this purpose, different colorimetric techniques such as transmission spectrophotometry, diffuse reflectance spectrophotometry and spectroradiometry were applied. The samples consisted of increasing dilutions $(0,20,40,60,80$ and $100 \%)$ of the phenolic fractions obtained from 4 wines: Tempranillo (T) and Graciano $(G)$ monovarietal wines, and two 80:20 mixtures: $M$ (wine elaborated by blending grapes) and W (a blend of the $T$ and $G$ wines) ( 9 fractions per wine). Fractionation was performed using gel permeation chromatography with a Toyopearl HW-40S column, and the dilutions of the fractions were performed with synthetic wine $(\mathrm{pH}=3.6)$. The spectroradiometric measurements permitted the differences due to the dilution effect on the fractions to be established more clearly than with the results obtained using diffuse reflectance and spectrophotometry. Thus, this technology is very suitable for use in comparative interpretations by the human eye. In turn, we assessed the changes in colour due to the effect of dilution on the fractions, observing that the effect of dilution led to an increase in the values of lightness $\left(\mathrm{L}^{*}\right)$, while the chroma values $\left(\mathrm{C}^{*} \mathrm{ab}\right)$ followed the opposite trend, in agreement with its role as a variable related to chromatic intensity or vividness of the sample. In contrast, hue $\left(h_{a b}\right)$ did not seem to be affected by dilution of the fractions, in consonance with the qualitative nature of this parameter.
\end{abstract}

(c) 2012 Elsevier B.V. All rights reserved.

\section{Introduction}

In the field of oenology, the visual analysis of wine colour is included in the tasting or organoleptic examination, subjecting the wine to our senses to determine its sensory characteristics, eventually appreciating it (or not) [1]. In red wines, colour represents the first sensory characteristic perceived by the taster, providing not only information about defects, type or the evolution of the wine, but also greatly influence on its acceptability [2] and price [3]. So, strong correlations have been found between the colour and overall quality of wines $[4,5]$.

The initial violet-red colour of young red wines is the net result of all the monomer, oligomer and polymer anthocyanins extracted from the grape skins, together with their copigmented forms and intensity and hue, dependent upon factors such as the nature and concentration of the individual anthocyanins and their degree of

\footnotetext{
* Corresponding author. Tel.: +34 954556495; fax: +34 954557017.

E-mail address: heredia@us.es (F.J. Heredia).
}

degradation, temperature, $\mathrm{pH}$, the nature of the solvent, the presence of $\mathrm{SO}_{2}$, oxygen, enzymes, copigments, sugars, etc. [6-12]. However, during the ageing process of wines this colour evolves to reddish-orange hues, mainly due to the progressive structural changes undergone by the anthocyanins. These changes occur through different mechanisms [7,13-18].

In the winery, the parameters traditionally used to describe the variation in colour of the anthocyanin solutions have mainly been the changes in $\lambda_{\max }$ in the visible part of the spectrum as a measurement of variations in hue, together with changes in absorbance for the variation in colour intensity $[19,20]$. Both indices are easy to calculate and interpret and are those most frequently used in winery [21]. Nevertheless, Gonnet [22] reported that an adequate description of variations in the colour of anthocyanin solutions, originated, for example, by $\mathrm{pH}$, require the following: (a) that the spectral variations considered should be those affecting the whole spectral curve, not only its visible $\lambda_{\max }$; (b) that it would be appropriate to use the three colour attributes (hue, saturation and lightness) for its description, and (c) that these should refer to the conditions of the observer and of the light source. 
<smiles>[2H]c1cc(-c2[o+]c3cc(O)cc(O)c3cc2OC2O[C@H](CC)[C@H](O)[C@H](O)[C@H]2O)cc(Br)c1O</smiles>

Hydroxilation and Methoxylation patterns

Name

Cyanidin (Cy)

Delphinidin (Dp)

Peonidin (Pn)

Petunidin (Pt)

Malvidin (Mv)
$\mathrm{R}_{1} \quad \mathrm{R}_{2}$

$\mathrm{OH} \quad \mathrm{H}$

$\mathrm{OH} \quad \mathrm{OH}$

$\mathrm{OCH}_{3} \quad \mathrm{H}$

$\begin{array}{lll}\mathrm{OCH}_{3} & \mathrm{OH} & \text { Acylation patterns }\end{array}$

$\mathrm{OCH}_{3} \quad \mathrm{OCH}_{3}$
$\mathrm{R}_{3}$

$\mathrm{OH}$<smiles>CCOC(=O)/C=C/c1ccc(O)cc1</smiles>

A

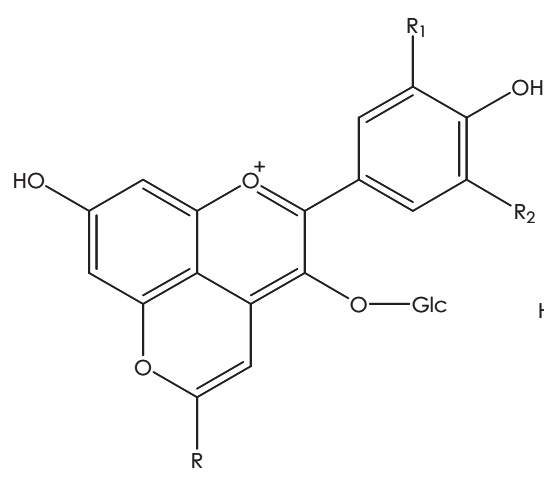

B

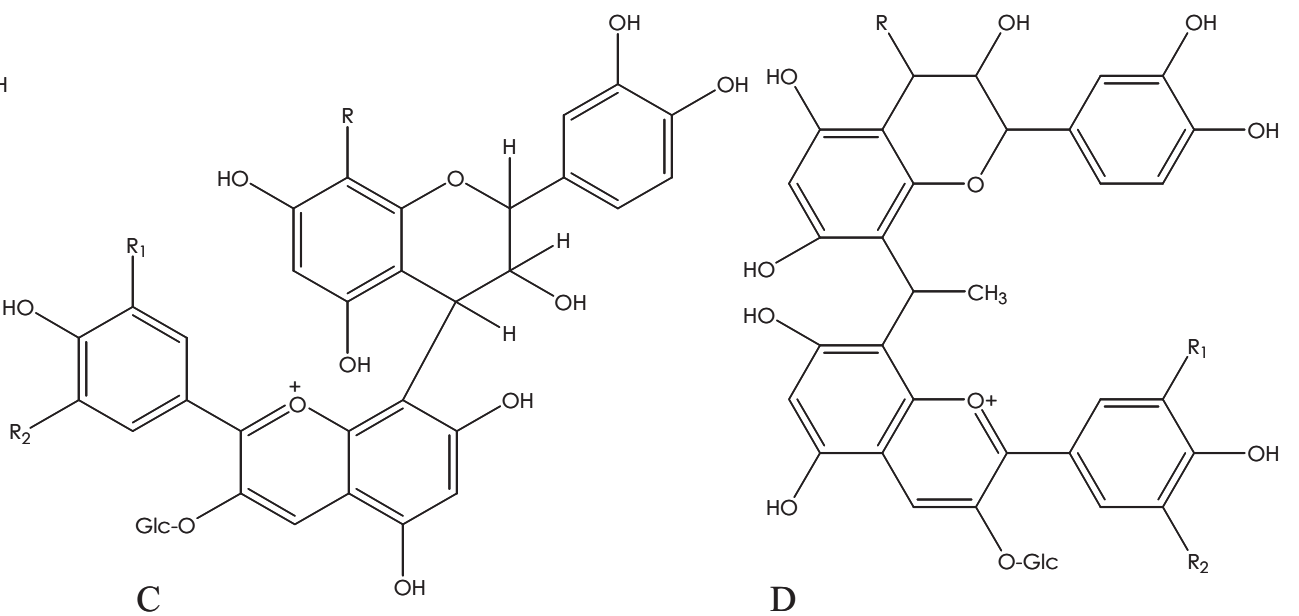

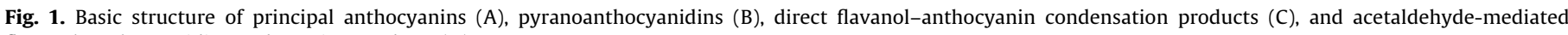
flavanol-anthocyanidin condensation products (D).

The Commission Internationale de l'Eclairage (CIE) has proposed different systems for colour representation in an attempt to find one that will reflect the visual sensation perceived by observers in an appropriate way. When the determination of a colour is carried out it is necessary to determine the position of the observer, the light source, and the interval of data acquisition.

The colour of objects can be expressed through the colour coordinates of the different colour spaces [23]. The CIE $1976\left(L^{*}, a^{*}\right.$, and $b^{*}$ ) or CIELAB colour space is a Cartesian coordinate system defined by three colorimetric coordinates $L^{*}, a^{*}$, and $b^{*}$. $L^{*}$ represents lightness, taking values from 0 (black) to 100 (white). The coordinate $a^{*}$ takes positive values for reddish colours and negative values for greenish ones, whilst $b^{*}$ is positive for yellowish colours and negative for the bluish ones. From these three coordinates, other colour parameters are defined within this space: hue $\left(h_{a b}\right)$, the angular parameter considered the qualitative attribute of colourfulness, and chroma $\left(\mathrm{C}^{*}{ }_{\mathrm{ab}}\right)$, the quantitative attribute of colourfulness which can be used to assess the degree of difference of any hue relative to a grey colour with the same lightness.

Generally, the colour of pure anthocyanins solutions has been measured by transmission spectrophotometry within the CIELAB colour space [22,24-29]. Reflectance measurements have been applied to characterise the colour of translucent foods, orange juices and honeys [30,31].

The aim of this work was to study the influence of the dilution effect on the colour of the fractions and to evaluate the relationship between the colour of the phenolic fractions of red wines containing pigments, measured by transmission and reflection techniques, and to determine which of these techniques allows a better interpretation of the colour to be obtained.

\section{Materials and methods}

\subsection{Winemaking and samples}

Vitis vinifera cv. Tempranillo and Graciano fresh grapes from the Spanish D.O. La Rioja, were collected by Bodegas Roda S.A at the optimum maturation level, from different randomly selected vines and from different parts of several clusters. A portion of each sample was immediately analysed for oenological parameters. With the remainder of grapes three wines were elaborated separately: $T$ from the Tempranillo variety, $G$ from the Graciano variety, and $\mathrm{M}$ from 80:20 mixture of Tempranillo and Graciano grapes. A fourth wine $W$ was elaborated by blending $(80: 20, v / v)$ the $T$ and $\mathrm{G}$ wines after end of malolactic fermentation. For the wine elaboration, grapes were crushed and destemmed and the mass was put into $1200 \mathrm{~L}$ stainless steel vats. After pre-fermentative maceration stage (2-4 days at $14{ }^{\circ} \mathrm{C}$ ) the alcoholic fermentation (must together to skins and seeds) occurred for 7-12 days. Then, it was a period of post-fermentative maceration (5-7 days) and after this, wines were transferred to 225 L French oak barrels where the malolactic fermentation occurred $\left(21^{\circ} \mathrm{C}\right.$ during approximately 30 days $)$ and the ageing process started at $9-12^{\circ} \mathrm{C}$. 


\subsection{Enological parameters}

Sugar content, $\mathrm{pH}$ and titratable acidity in grape samples were determined using the International Organisation of Vine and Wine (OIV) methods [32]. Anthocyanins were determined by the Ribereau-Gayon and Stonestreet method [33], and total polyphenolic index was determined by UV absorption at $280 \mathrm{~nm}$ [34].

\subsection{Sample fractionation}

After three months of ageing in a barrel, $180 \mathrm{~mL}$ aliquots of each wine sample (T, G, M and W wines) were collected and fractioned with a Toyopearl HW-40(s) gel column (Tosoh, Japan) [35]. The elution solvents were ethanol $/ \mathrm{H}_{2} \mathrm{O}(80: 20, \mathrm{v} / \mathrm{v})$ and methanol $/ \mathrm{H}_{2} \mathrm{O}$ $(80: 20, v / v)$. The different coloured bands formed during elution as well as the bleaching eluates were collected separately. Thus, nine fractions were obtained, dependent upon the change in colour produced inside the chromatographic column, each considered as a different family of pigments according to the major compounds present (Fig. 1). All fractions were acidified to $\mathrm{pH}=1$ in order to reverse the existing bisulphite-anthocyanin adducts, concentrated under vacuum, re-dissolved in water, and freeze-dried. Solutions of the freeze-dried fractions were prepared to have similar contents as in the wines. Thus, depending on the fraction different amounts

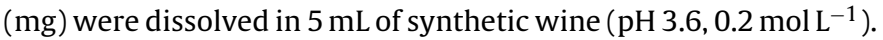

\subsection{HPLC-DAD-MS analysis}

The solutions of the fractions were acidified with acidic water (pH 3.6) (Panreac ${ }^{\circledR}$ Barcelona, Spain) and injected into the chromatographic system after filtration through a $0.45 \mu \mathrm{m}$ Millex ${ }^{\circledR}$ syringe-driven filter unit (Millipore Corporation).

HPLC-DAD analysis was performed with a Hewlett-Packard 1100 series liquid chromatograph. The LC system was connected to the probe of the mass spectrometer via the UV cell outlet. The mass analyses were performed using a Finnigan ${ }^{\mathrm{TM}}$ LCQ ion trap detector (Thermoquest, San Jose, CA, USA) equipped with an API source, using an electrospray ionisation (ESI) interface. The HPLCDAD-MS analysis of red pigments was carried out in accordance with García-Marino et al. [36].

\subsection{Quantification}

For quantitative analyses, calibration curves were obtained using standards of anthocyanin 3-O-glucosides (delphinidin 3$O$-glucoside, cyanidin 3-O-glucoside, petunidin 3-O-glucoside, peonidin 3-O-glucoside and malvidin 3-O-glucoside). Anthocyanins were purchased from Polyphenols Labs., Sandnes, Norway.

All pigments were quantified from the areas of their chromatographic peaks at $520 \mathrm{~nm}$, and the results were expressed in $\mathrm{mg} \mathrm{L}^{-1}$

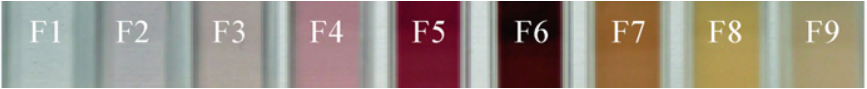

Fig. 2. The nine pigment fractions obtained from red wines (an example).

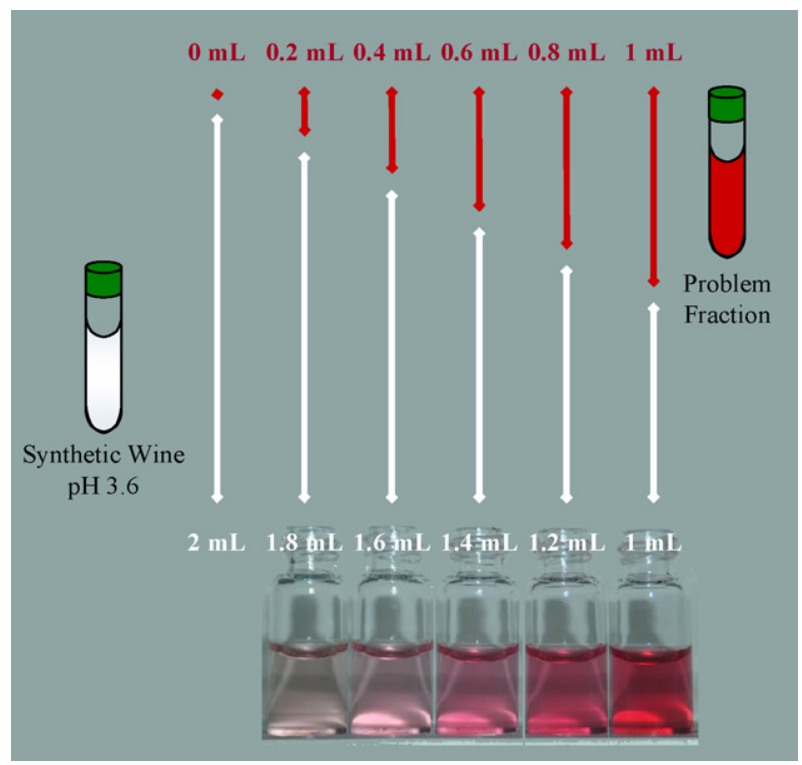

Fig. 3. Preparation of dilutions $(0,20,40,60,80$, and $100 \%)$ for the dilution effect assays.

of wine. The total content of the different groups of pigments studied was calculated from the sum of the individual concentrations obtained for each individual compound.

\subsection{Colorimetric measurements}

Prior to spectrophotometric analysis, the fractions were filtered through Millipore-AP20 filters (Millipore Corporation, Bedford, MA, USA). Plastic cells $(475 \mathrm{~mm} \times 350 \mathrm{~mm} \times 10 \mathrm{~mm})$ were used for the measurements.

Three colorimetric techniques were applied, one based on light transmission and two based on light reflection.

Transmission measurements, specific for transparent samples, were made with a UV/visible HP8452 (Hewlett-Packard, Palo Alto, CA, USA) spectrophotometer diode-array. The whole visible spectra were recorded $(380-780 \mathrm{~nm}, \Delta \lambda=2 \mathrm{~nm})$. The CIE- $196410^{\circ}$ standard observer and CIE D65 standard illuminant (corresponding to day light) were taken as references to calculate the tristimulus values recommended by the Comission Internationale de l'Éclairage (CIE, 2004) by applying CromaLab software [37].

Table 1

Enological parameters determined in Tempranillo and Graciano grapes at harvest, as well as in T, G, and M wines just after alcoholic fermentation.

\begin{tabular}{|c|c|c|c|c|c|}
\hline \multirow[t]{2}{*}{ Enological parameters } & \multicolumn{2}{|c|}{ Grapes (harvest) } & \multicolumn{3}{|c|}{ Wines (end of alcoholic fermentation) } \\
\hline & Tempranillo & Graciano & $\mathrm{T}$ & G & $\mathrm{M}$ \\
\hline Date & 04-October & 03-October & 24-October & & \\
\hline Total acidity & 3.4 & 5.2 & 3.9 & 4.6 & 4.2 \\
\hline $\mathrm{pH}$ & 3.3 & 3.1 & 4.0 & 3.5 & 3.8 \\
\hline Brix grade $\left({ }^{\circ} \mathrm{Bx}\right)$ & 13.4 & 14.2 & 13.6 & 14.1 & 13.2 \\
\hline Absrobance $420 \mathrm{~nm}$ & 0.5 & 0.7 & 0.7 & 1.0 & 0.7 \\
\hline Absrobance $520 \mathrm{~nm}$ & 1.1 & 0.2 & 1.5 & 2.4 & 1.6 \\
\hline Absrobance $620 \mathrm{~nm}$ & 0.2 & 0.3 & 0.3 & 0.4 & 0.3 \\
\hline Colour index & 18.2 & 28.5 & 25.4 & 37.3 & 25.7 \\
\hline Berry weight (g) & 1.6 & 1.0 & - & - & - \\
\hline Total anthocyanins ( $\mathrm{mg} \mathrm{L}^{-1}$ in wine) & - & - & 1007.5 & 1246.7 & 1040.0 \\
\hline Total polyphenol index & - & - & 85.8 & 84.3 & 81.0 \\
\hline
\end{tabular}


Table 2

Mean concentration ( $\mathrm{mg} \mathrm{L}^{-1}, \pm$ S.D.; $n=3$ ) of different pigment families of the fraction solutions (1-9) of the $\mathrm{T}, \mathrm{G}, \mathrm{M}$ and $\mathrm{W}$ wines.

\begin{tabular}{|c|c|c|c|c|c|c|c|c|c|}
\hline \multirow[t]{3}{*}{ Fraction } & \multicolumn{9}{|l|}{ Pigments } \\
\hline & $\begin{array}{l}\text { Total } \\
\text { anthocyamdin- } \\
\text { monoglucosides }\end{array}$ & $\begin{array}{l}\text { Total } \\
\text { anthocyamdin- } \\
\text { diglucosides }\end{array}$ & $\begin{array}{l}\text { Total acylated } \\
\text { anthocyanins }\end{array}$ & $\begin{array}{l}\text { Total } \\
\text { anthocyanins }\end{array}$ & $\begin{array}{l}\text { Total } \\
\text { pyranoanthocyanidins }\end{array}$ & $\begin{array}{l}\text { Acetaldehy } \\
\text { de-mediated flavanol- } \\
\text { anthocyanidin } \\
\text { condensation products }\end{array}$ & $\begin{array}{l}\text { Direct flavanol- } \\
\text { anthocyanin } \\
\text { condensation } \\
\text { products }\end{array}$ & $\begin{array}{l}\text { Total derived } \\
\text { pigments }\end{array}$ & Total pigments \\
\hline & Mean \pm S.D. & Mean \pm S.D. & Mean \pm S.D. & Mean \pm S.D. & Mean \pm S.D. & Mean \pm S.D. & Mean \pm S.D. & Mean \pm S.D. & Mean \pm S.D. \\
\hline \multicolumn{10}{|c|}{ Wine: Tempranillo ( $\mathrm{T}$ ) } \\
\hline 1 & $4.16 \pm 0.00$ & $0.00 \pm 0.00$ & $0.00 \pm 0.00$ & $4.16 \pm 0.00$ & $0.00 \pm 0.00$ & $0.00 \pm 0.00$ & $0.00 \pm 0.00$ & $0.00 \pm 0.00$ & $4.16 \pm 0.00$ \\
\hline 2 & $18.93 \pm 0.04$ & $6.67 \pm 0.00$ & $6.84 \pm 0.00$ & $32.44 \pm 0.05$ & $0.00 \pm 0.00$ & $2.47 \pm 0.00$ & $5.94 \pm 0.00$ & $8.41 \pm 0.00$ & $40.85 \pm 0.05$ \\
\hline 3 & $32.04 \pm 0.16$ & $0.00 \pm 0.00$ & $34.11 \pm 0.06$ & $66.14 \pm 0.23$ & $8.03 \pm 0.01$ & $3.08 \pm 0.01$ & $0.00 \pm 0.00$ & $11.11 \pm 0.03$ & $77.25 \pm 0.26$ \\
\hline 4 & $38.00 \pm 0.24$ & $0.00 \pm 0.00$ & $33.45 \pm 0.08$ & $71.45 \pm 0.32$ & $9.52 \pm 0.02$ & $2.79 \pm 0.00$ & $0.00 \pm 0.00$ & $12.31 \pm 0.05$ & $83.76 \pm 0.36$ \\
\hline 5 & $110.84 \pm 0.95$ & $15.22 \pm 0.05$ & $46.59 \pm 0.16$ & $172.65 \pm 1.16$ & $18.32 \pm 0.07$ & $3.45 \pm 0.01$ & $0.00 \pm 0.00$ & $21.77 \pm 0.16$ & $194.41 \pm 1.33$ \\
\hline 6 & $95.93 \pm 0.77$ & $13.03 \pm 0.02$ & $60.04 \pm 0.26$ & $169.01 \pm 1.05$ & $37.35 \pm 0.19$ & $3.83 \pm 0.01$ & $10.87 \pm 0.01$ & $52.73 \pm 0.44$ & $221.04 \pm 1.49$ \\
\hline 7 & $17.79 \pm 0.03$ & $7.77 \pm 0.00$ & $18.03 \pm 0.01$ & $43.59 \pm 0.04$ & $8.60 \pm 0.00$ & $2.55 \pm 0.00$ & $14.78 \pm 0.01$ & $25.93 \pm 0.04$ & $69.51 \pm 0.08$ \\
\hline 8 & $0.00 \pm 0.00$ & $0.00 \pm 0.00$ & $0.00 \pm 0.00$ & $\mathbf{0 . 0 0} \pm \mathbf{0 . 0 0}$ & $0.00 \pm 0.00$ & $0.00 \pm 0.00$ & $0.00 \pm 0.00$ & $\mathbf{0 . 0 0} \pm \mathbf{0 . 0 0}$ & $0.00 \pm 0.00$ \\
\hline 9 & $2.79 \pm 0.00$ & $0.00 \pm 0.00$ & $0.00 \pm 0.00$ & $2.79 \pm 0.00$ & $0.00 \pm 0.00$ & $0.00 \pm 0.00$ & $0.00 \pm 0.00$ & $0.00 \pm 0.00$ & $2.79 \pm 0.00$ \\
\hline Total & $320.48 \pm 2.19 \mathrm{a} / \mathrm{A}$ & $42.69 \pm 0.07 \mathrm{~b} / \mathrm{B}$ & $199.06 \pm 0.57 \mathrm{c} / \mathrm{B}$ & $562.23 \pm 2.85 \mathrm{a} / \mathrm{A}$ & $81.82 \pm 0.29 a / B$ & $18.17 \pm 0.03 \mathrm{~d} / \mathrm{D}$ & $31.59 \pm 0.02 \mathrm{c} / \mathrm{D}$ & $132.26 \pm 0.72 \mathrm{a} / \mathrm{B}$ & $693.77 \pm 3.57 \mathrm{a} / \mathrm{A}$ \\
\hline \multicolumn{10}{|c|}{ Wine: Graciano (G) } \\
\hline 1 & $9.74 \pm 0.00$ & $0.00 \pm 0.00$ & $2.46 \pm 0.00$ & $\mathbf{1 2 . 2 0} \pm \mathbf{0 . 0 0}$ & $0.00 \pm 0.00$ & $0.00 \pm 0.00$ & $0.00 \pm 0.00$ & $0.00 \pm 0.00$ & $12.20 \pm 0.00$ \\
\hline 2 & $26.11 \pm 0.12$ & $10.44 \pm 0.01$ & $24.32 \pm 0.01$ & $60.87 \pm 0.13$ & $1.53 \pm 0.00$ & $2.56 \pm 0.00$ & $6.03 \pm 0.00$ & $10.12 \pm 0.01$ & $70.99 \pm 0.14$ \\
\hline 3 & $140.65 \pm 1.27$ & $11.07 \pm 0.01$ & $41.29 \pm 0.11$ & $193.02 \pm 1.40$ & $11.49 \pm 0.01$ & $3.09 \pm 0.01$ & $3.46 \pm 0.00$ & $18.05 \pm 0.04$ & $211.06 \pm 1.44$ \\
\hline 4 & $105.98 \pm 0.92$ & $14.59 \pm 0.05$ & $42.86 \pm 0.16$ & $163.43 \pm 1.13$ & $24.52 \pm 0.09$ & $0.00 \pm 0.00$ & $0.00 \pm 0.00$ & $24.52 \pm 0.17$ & $187.95 \pm 1.31$ \\
\hline 5 & $82.06 \pm 0.63$ & $15.74 \pm 0.06$ & $54.58 \pm 0.24$ & $152.39 \pm 0.93$ & $31.26 \pm 0.12$ & $3.40 \pm 0.01$ & $2.53 \pm 0.00$ & $37.20 \pm \mathbf{0 . 2 6}$ & $189.59 \pm 1.19$ \\
\hline 6 & $49.53 \pm 0.30$ & $12.36 \pm 0.01$ & $42.38 \pm 0.08$ & $104.26 \pm 0.39$ & $21.98 \pm 0.06$ & $2.89 \pm 0.00$ & $7.16 \pm 0.01$ & $32.03 \pm 0.16$ & $136.31 \pm 0.55$ \\
\hline 7 & $19.51 \pm 0.04$ & $4.14 \pm 0.00$ & $22.59 \pm 0.01$ & $46.24 \pm 0.05$ & $2.57 \pm 0.00$ & $2.55 \pm 0.00$ & $15.02 \pm 0.02$ & $20.14 \pm 0.04$ & $66.38 \pm 0.08$ \\
\hline 8 & $0.00 \pm 0.00$ & $0.00 \pm 0.00$ & $0.00 \pm 0.00$ & $\mathbf{0 . 0 0} \pm \mathbf{0 . 0 0}$ & $0.00 \pm 0.00$ & $0.00 \pm 0.00$ & $0.00 \pm 0.00$ & $\mathbf{0 . 0 0} \pm \mathbf{0 . 0 0}$ & $0.00 \pm 0.00$ \\
\hline 9 & $0.00 \pm 0.00$ & $0.00 \pm 0.00$ & $0.00 \pm 0.00$ & $0.00 \pm 0.00$ & $0.00 \pm 0.00$ & $0.00 \pm 0.00$ & $0.00 \pm 0.00$ & $0.00 \pm 0.00$ & $0.00 \pm 0.00$ \\
\hline Total & $433.58 \pm 3.28 \mathrm{c} / \mathrm{A}$ & $68.34 \pm 0.14 \mathrm{~d} / \mathrm{D}$ & $230.48 \pm 0.61 \mathrm{~d} / \mathrm{C}$ & $732.41 \pm 4.03 \mathrm{c} / \mathrm{A}$ & $93.35 \pm 0.28 \mathrm{c} / \mathrm{B}$ & $14.49 \pm 0.02 \mathrm{c} / \mathrm{C}$ & $34.20 \pm 0.03 \mathrm{~d} / \mathrm{D}$ & $142.06 \pm 0.68 \mathrm{~b} / \mathrm{B}$ & $874.48 \pm 4.71 \mathrm{c} / \mathrm{A}$ \\
\hline \multicolumn{10}{|c|}{ Wine: Blend of grapes $(\mathrm{M})$} \\
\hline 1 & $4.23 \pm 0.00$ & $0.00 \pm 0.00$ & $0.00 \pm 0.00$ & $4.23 \pm 0.00$ & $0.00 \pm 0.00$ & $0.00 \pm 0.00$ & $0.00 \pm 0.00$ & $0.00 \pm 0.00$ & $4.23 \pm 0.00$ \\
\hline 2 & $18.33 \pm 0.04$ & $4.10 \pm 0.00$ & $4.12 \pm 0.00$ & $26.56 \pm 0.04$ & $1.20 \pm 0.00$ & $0.00 \pm 0.00$ & $3.46 \pm 0.00$ & $4.65 \pm 0.00$ & $31.21 \pm 0.04$ \\
\hline 3 & $36.15 \pm 0.22$ & $0.00 \pm 0.00$ & $26.13 \pm 0.05$ & $62.28 \pm 0.27$ & $11.54 \pm 0.01$ & $2.81 \pm 0.00$ & $3.45 \pm 0.00$ & $17.81 \pm 0.03$ & $80.10 \pm 0.30$ \\
\hline 4 & $43.01 \pm 0.29$ & $6.21 \pm 0.00$ & $35.38 \pm 0.10$ & $84.60 \pm 0.39$ & $12.41 \pm 0.01$ & $2.60 \pm 0.00$ & $0.00 \pm 0.00$ & $15.02 \pm 0.03$ & $99.62 \pm 0.42$ \\
\hline 5 & $233.42 \pm 2.16$ & $25.14 \pm 0.11$ & $65.68 \pm 0.38$ & $324.24 \pm 2.65$ & $46.77 \pm 0.30$ & $3.84 \pm 0.01$ & $2.68 \pm 0.00$ & $53.29 \pm 0.63$ & $377.53 \pm 3.29$ \\
\hline 6 & $28.27 \pm 0.14$ & $15.12 \pm 0.01$ & $24.50 \pm 0.02$ & $67.89 \pm 0.17$ & $14.74 \pm 0.01$ & $2.49 \pm 0.00$ & $10.42 \pm 0.01$ & $27.63 \pm 0.05$ & $95.52 \pm 0.22$ \\
\hline 7 & $14.51 \pm 0.04$ & $6.65 \pm 0.00$ & $16.13 \pm 0.00$ & $37.29 \pm \mathbf{0 . 0 4}$ & $2.50 \pm 0.00$ & $2.48 \pm 0.00$ & $10.34 \pm 0.01$ & $15.32 \pm 0.02$ & $52.61 \pm 0.06$ \\
\hline 8 & $2.65 \pm 0.00$ & $0.00 \pm 0.00$ & $0.00 \pm 0.00$ & $2.65 \pm 0.00$ & $0.00 \pm 0.00$ & $0.00 \pm 0.00$ & $0.00 \pm 0.00$ & $0.00 \pm 0.00$ & $2.65 \pm 0.00$ \\
\hline 9 & $2.56 \pm 0.00$ & $0.00 \pm 0.00$ & $0.00 \pm 0.00$ & $2.56 \pm 0.00$ & $0.00 \pm 0.00$ & $0.00 \pm 0.00$ & $0.00 \pm 0.00$ & $0.00 \pm 0.00$ & $2.56 \pm 0.00$ \\
\hline Total & $383.13 \pm 2.89 \mathrm{~b} / \mathrm{A}$ & $57.22 \pm 0.12 c / C$ & $171.94 \pm 0.55 \mathrm{a} / \mathrm{A}$ & $612.30 \pm 3.56 \mathrm{~b} / \mathrm{B}$ & $89.16 \pm 0.33 \mathrm{~b} / \mathrm{D}$ & $14.22 \pm 0.01 \mathrm{~b} / \mathrm{D}$ & $30.35 \pm 0.02 \mathrm{~b} / \mathrm{C}$ & $\mathbf{1 3 3 . 7 2} \pm \mathbf{0 . 7 6} \mathrm{a} / \mathrm{D}$ & $746.03 \pm 4.33 \mathrm{~b} / \mathrm{B}$ \\
\hline \multicolumn{10}{|c|}{ Wine: Blend of wines (W) } \\
\hline 1 & $0.00 \pm 0.00$ & $0.00 \pm 0.00$ & $0.00 \pm 0.00$ & $0.00 \pm 0.00$ & $0.00 \pm 0.00$ & $0.00 \pm 0.00$ & $0.00 \pm 0.00$ & $0.00 \pm 0.00$ & $0.00 \pm 0.00$ \\
\hline 2 & $21.24 \pm 0.07$ & $4.19 \pm 0.00$ & $17.86 \pm 0.01$ & $43.29 \pm 0.08$ & $18.19 \pm 0.02$ & $2.51 \pm 0.00$ & $3.56 \pm 0.00$ & $24.26 \pm 0.05$ & $67.55 \pm 0.13$ \\
\hline 3 & $19.97 \pm 0.05$ & $0.00 \pm 0.00$ & $23.35 \pm 0.01$ & $43.32 \pm 0.07$ & $9.25 \pm 0.00$ & $0.00 \pm 0.00$ & $0.00 \pm 0.00$ & $9.24 \pm 0.01$ & $52.57 \pm 0.08$ \\
\hline 4 & $122.00 \pm 1.06$ & $12.25 \pm 0.02$ & $48.48 \pm 0.17$ & $182.72 \pm 1.25$ & $31.89 \pm 0.11$ & $3.50 \pm 0.01$ & $3.50 \pm 0.00$ & $38.89 \pm 0.25$ & $221.63 \pm 1.50$ \\
\hline 5 & $187.35 \pm 1.70$ & $19.08 \pm 0.05$ & $60.44 \pm 0.30$ & $266.87 \pm 2.05$ & $33.89 \pm 0.19$ & $3.51 \pm 0.01$ & $8.74 \pm 0.02$ & $46.14 \pm 0.45$ & $313.01 \pm 2.49$ \\
\hline 6 & $18.71 \pm 0.04$ & $0.00 \pm 0.00$ & $14.80 \pm 0.00$ & $33.51 \pm \mathbf{0 . 0 5}$ & $10.13 \pm 0.01$ & $2.46 \pm 0.00$ & $3.85 \pm 0.00$ & $16.43 \pm 0.03$ & $49.94 \pm 0.08$ \\
\hline 7 & $13.19 \pm 0.02$ & $4.14 \pm 0.00$ & $16.02 \pm 0.00$ & $33.35 \pm 0.03$ & $2.48 \pm 0.00$ & $0.00 \pm 0.00$ & $6.54 \pm 0.01$ & $9.02 \pm 0.01$ & $42.37 \pm 0.04$ \\
\hline 8 & $2.69 \pm 0.00$ & $0.00 \pm 0.00$ & $0.00 \pm 0.00$ & $2.69 \pm 0.00$ & $0.00 \pm 0.00$ & $0.00 \pm 0.00$ & $0.00 \pm 0.00$ & $0.00 \pm 0.00$ & $2.69 \pm 0.00$ \\
\hline 9 & $0.00 \pm 0.00$ & $0.00 \pm 0.00$ & $0.00 \pm 0.00$ & $0.00 \pm 0.00$ & $0.00 \pm 0.00$ & $0.00 \pm 0.00$ & $0.00 \pm 0.00$ & $0.00 \pm 0.00$ & $0.00 \pm 0.00$ \\
\hline Total & $385.15 \pm 2.94 \mathrm{~b} / \mathrm{A}$ & $39.66 \pm 0.07 \mathrm{a} / \mathrm{A}$ & $180.95 \pm 0.49 \mathrm{~b} / \mathrm{A}$ & $605.75 \pm 3.53 \mathrm{~b} / \mathrm{A}$ & $105.83 \pm 0.33 \mathrm{~d} / \mathrm{B}$ & $11.98 \pm 0.02 \mathrm{a} / \mathrm{B}$ & $26.19 \pm 0.02 \mathrm{a} / \mathrm{A}$ & $143.98 \pm 0.80 \mathrm{~b} / \mathbf{B}$ & $749.76 \pm 4.32 \mathrm{~b} / \mathrm{A}$ \\
\hline
\end{tabular}

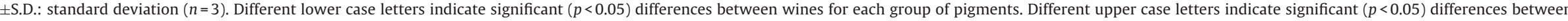
families of pigments for each wine. 
Tempranillo (T)

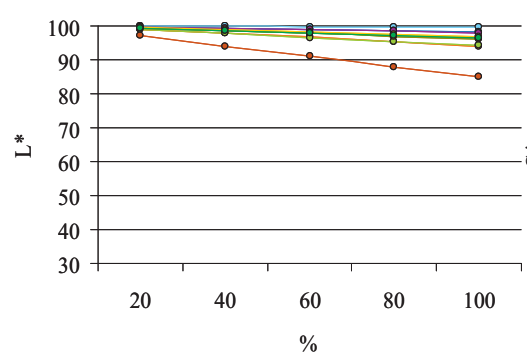

Graciano (G)

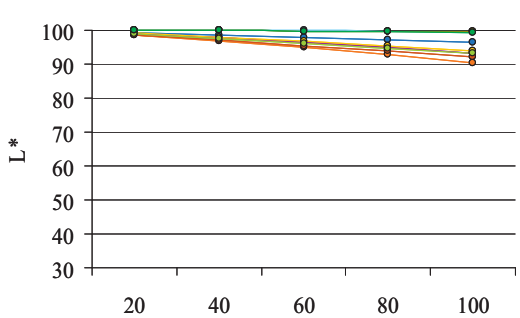

20
A.1.

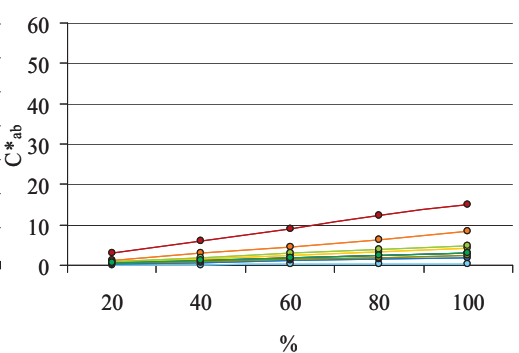

B.1.

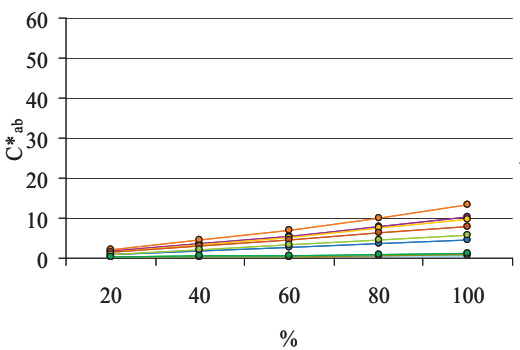

C.1.

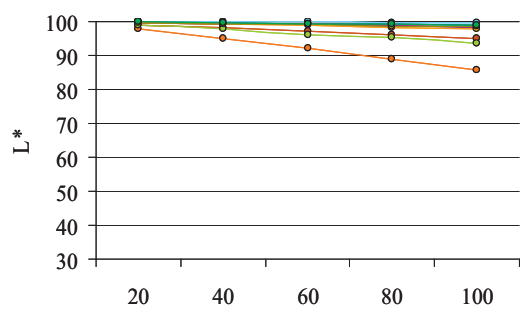

$20 \quad 40 \quad 60 \quad 80 \quad 100$
D.1

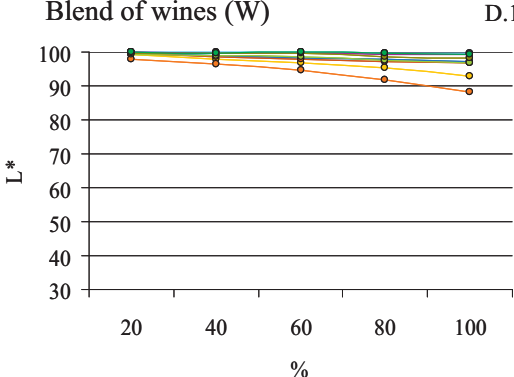

A.2.

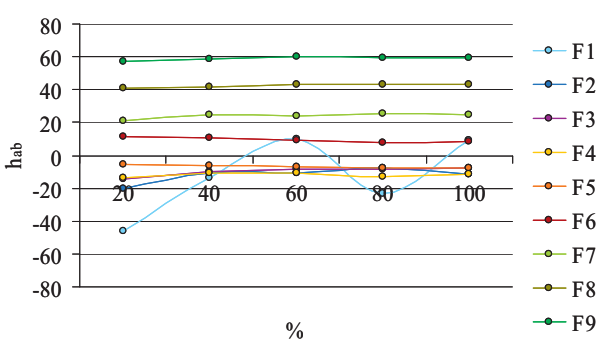

B.2.

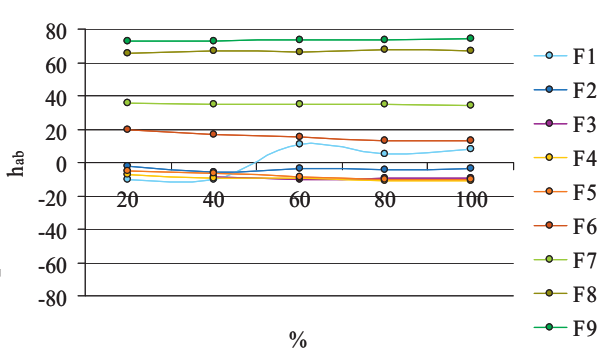

C.2.

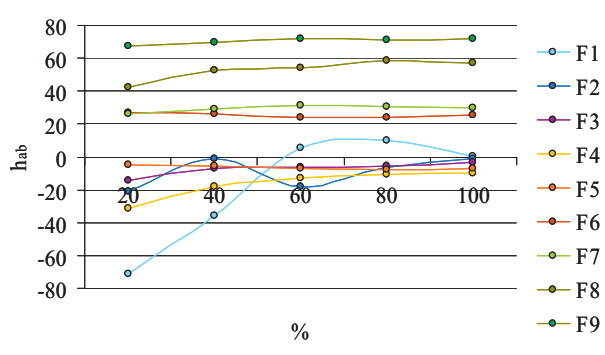

D.2.

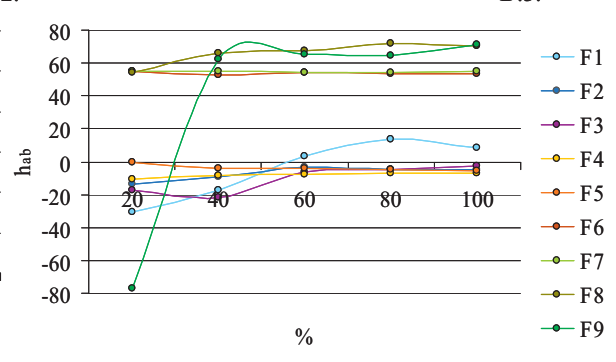

D.3.

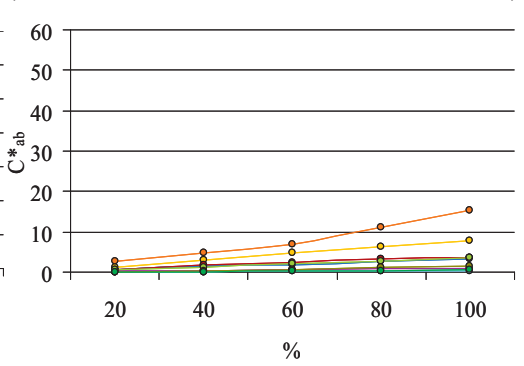

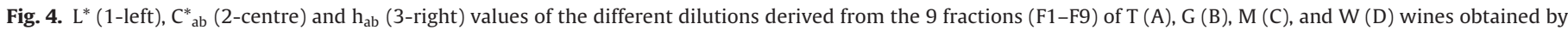
spectrophotometry. (For interpretation of the references to colour in this figure text, the reader is referred to the web version of the article.)

The reflectance measurements, which can be used for both transparent and translucent samples, were performed by spectroradiometry and diffuse reflectance spectrophotometry, based on the measurement of colour from the spectral radiance reflected by the object. In the case of spectroradiometry the light source is outside the instrument, and so samples are illuminated from a specific distance, just as the eye of a human observer does in real life (non-contact measurements), and for the diffuse reflectance the source is inside the apparatus (contact measurements). A CAS 140B (Instrument Systems, Munich, Germany) was used: coupled to spectroradiometer TOP 100 telescopic optical probe (Instrument Systems, Munich, Germany) and a Tamron SP 23A zoom (Tamron USA, Inc., Commack, NY, USA) for the spectroradiometric measurements, and connected to ISP80 integration sphere (Instrument Systems, Munich, Germany) for the diffuse reflectance measurements. In both cases samples were measured against white backing (pressed barium sulphate), the whole visible reflectance spectra were recorded ( $380-780 \mathrm{~nm}, \Delta \lambda=2 \mathrm{~nm}$ ) and the CIELAB parameters were calculated using IS-Specwin v.1.8.1.6 (Instrument Systems, Munich, Germany) software.

\subsection{Dilution assays pigment fractions}

Dilutions of fractions were assayed in order to study the influence of each dilution on the colour of the fractions and to generate a greater number of samples to allow the different colorimetric techniques to be compared. To accomplish this, increasing volumes of the target fraction were obtained (9 fractions obtained from the fractionation of each of the 4 wines studied) and were diluted in synthetic wine, $\mathrm{pH} 3.6$, finally obtaining mixtures with different percentages $(0,20,40,60,80$ and $100 \%)$, as shown in Fig. 2. The total number of fraction samples was 216; i.e., 54 per fractionated wine.

The colour changes due to the dilution effect were evaluated by the three techniques described above. 
Tempranillo (T)

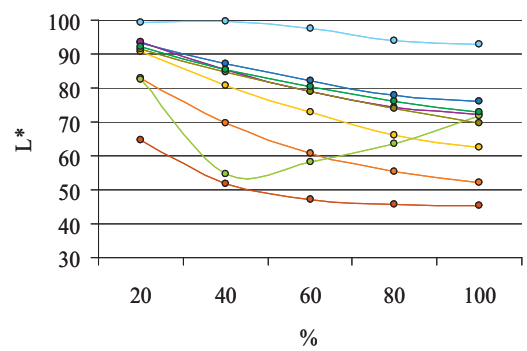

Graciano (G)

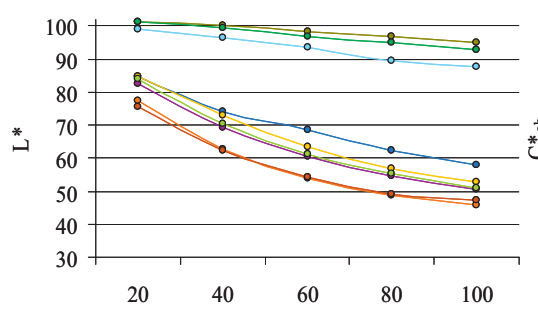

20

Blend of grapes (M)

C.1.

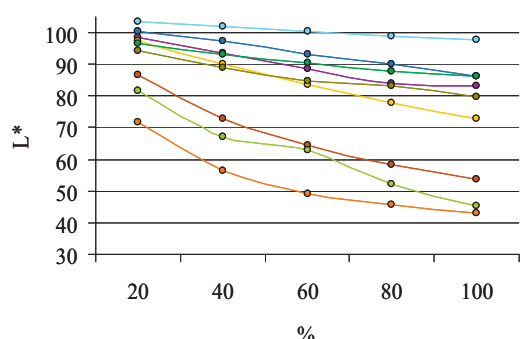

Blend of wines (W)

D.1.
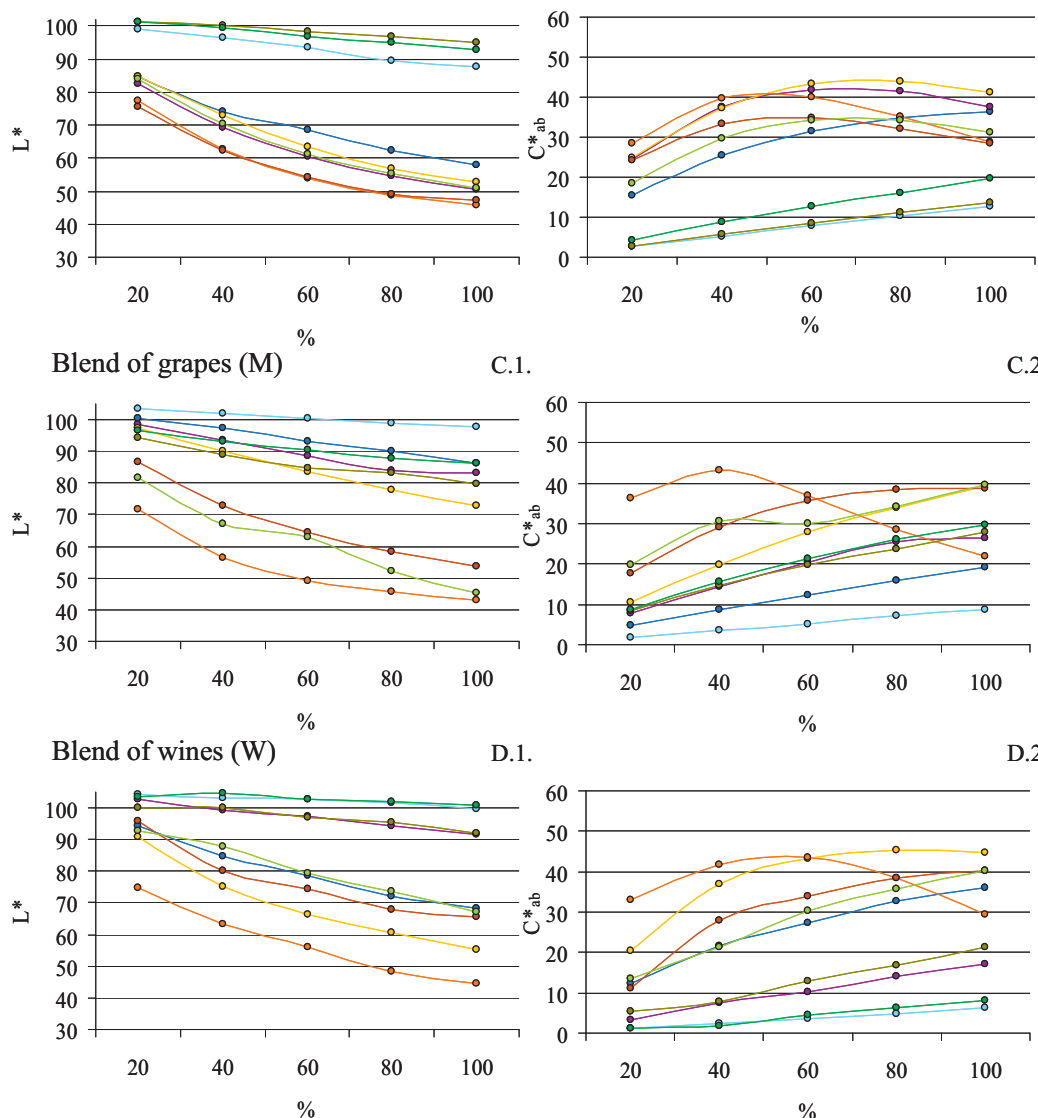

A.2.

A.3.

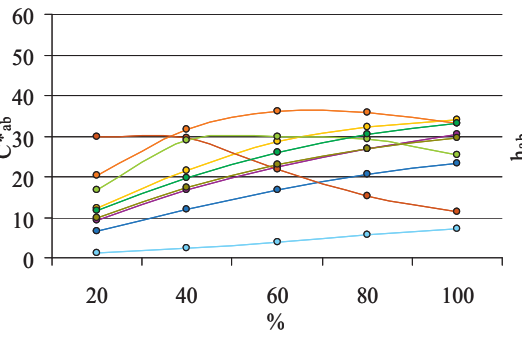

B.2.

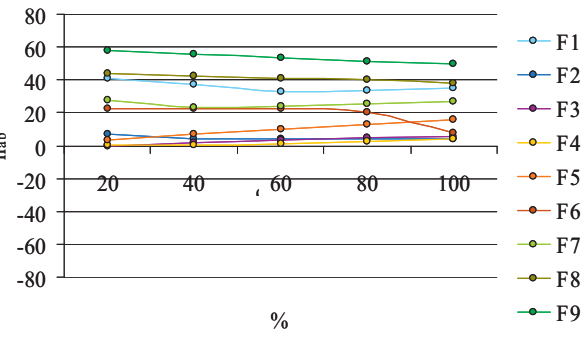

B.3.

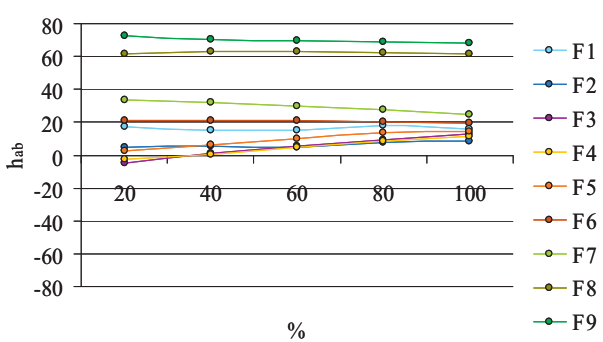

C.2.

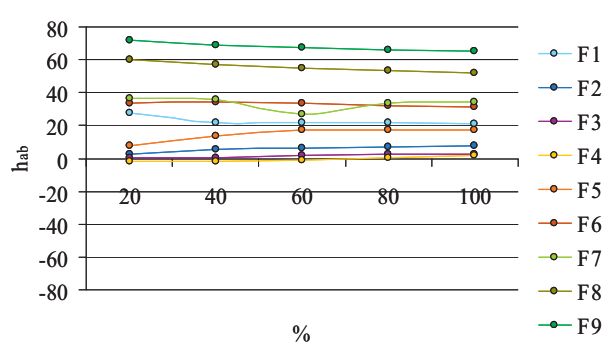

D.2.

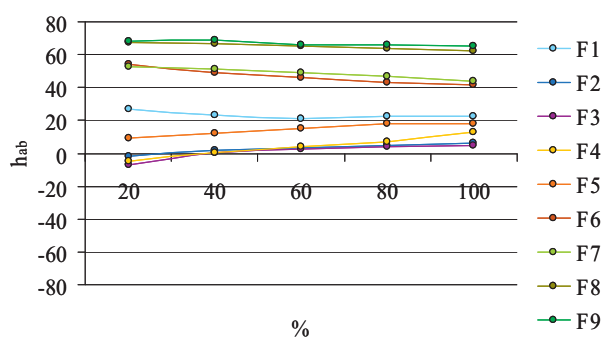

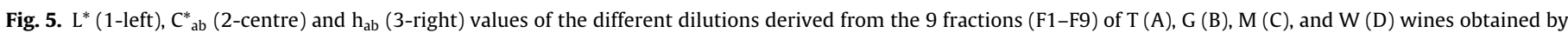
diffuse reflectance. (For interpretation of the references to colour in this figure text, the reader is referred to the web version of the article.)

\subsection{Statistical analyses}

Data are presented as means \pm standard deviations $( \pm$ S.D.) of three experiments performed in triplicate. Significant differences were determined by one-way analysis of variance (ANOVA) using an SPSS program, version 13.0, for Windows software package (SPSS, Inc., Chicago, IL).

\section{Results and discussion}

\subsection{Pigments in fractions}

Table 1 shows the enological parameters determined in Tempranillo and Graciano grapes at harvest as well as in T, G, and M wines just after alcoholic fermentation.

The use of mass spectrometry coupled to HPLC-DAD allowed the detection of thirty-seven anthocyanins and anthocyanin-derived pigments in the fraction samples: anthocyanidin-monoglucosides, anthocyanidin-diglucosides, acylated anthocyanins, pyranoanthocyanidins, acetaldehyde-mediated flavanol-anthocyanidin condensation products, and direct flavanol-anthocyanin condensation products (see Fig. 3). All the pigments identified in the fractions analysed have been described previously in samples of wines [36].

Table 2 shows the mean concentration of the different pigment families of the fraction solutions of the $T, G, M$ and $\mathrm{W}$ wines. It may be observed that the presence of anthocyanidin-monoglucosides was widespread in all fractions. The pigment contents were low in fractions 1,8 and 9. Furthermore, the anthocyanidin-monoglucoside compounds were the most abundant pigment family of the sum of total pigments in the nine fractions studied, followed by the acylated compounds, pyranoanthocyanidins and pigments derived from condensation between anthocyanins and flavanols (direct-linked and ethyllinked compounds), although these concentrations were different among the wines studied.

Analysis of variance (ANOVA) was carried out with the total pigment contents (obtained from the sum of the different fractions 
Tempranillo (T)

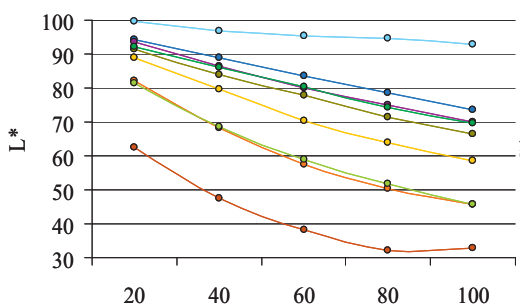

20
A.1.

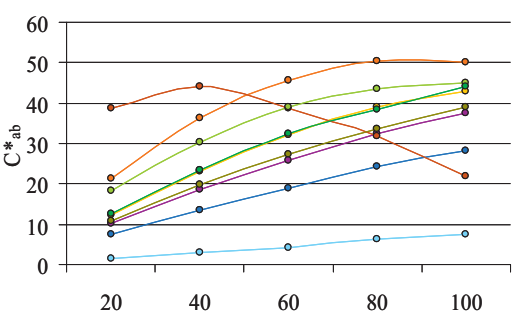

A. 2 .

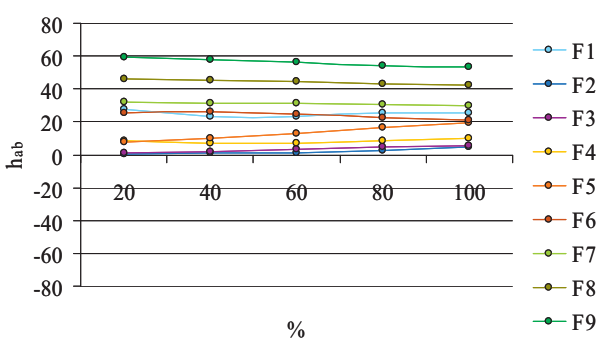

Graciano (G)

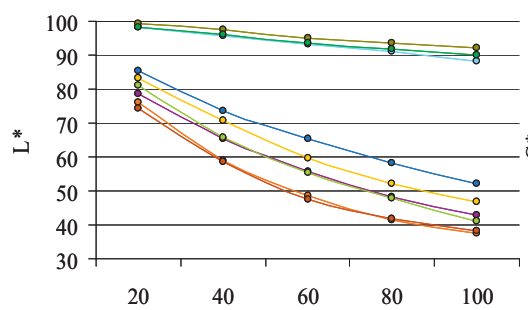

$20 \quad 40 \quad 60 \quad 80 \quad 100$

B.1.

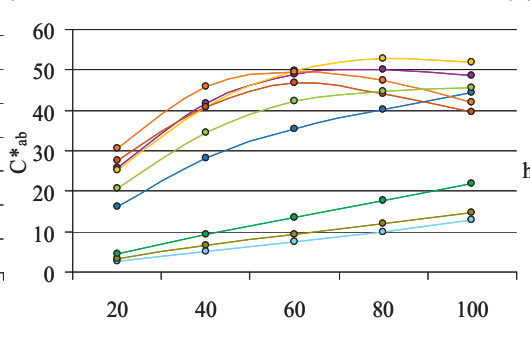

C.1.

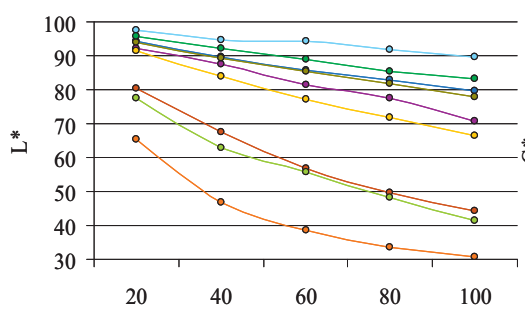

20 $\%$

Blend of wines (W)

D.1.

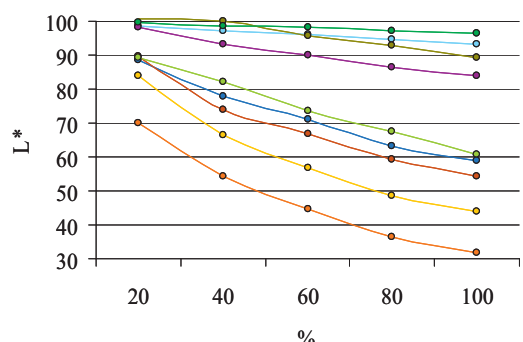

1.

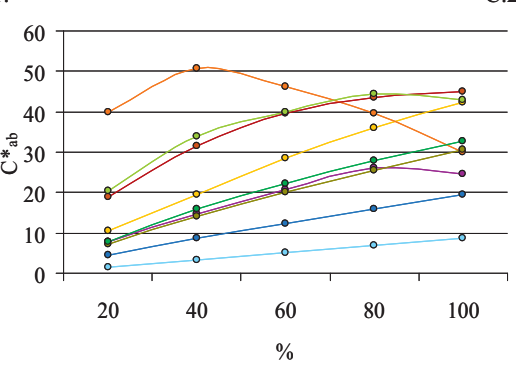

D.2.

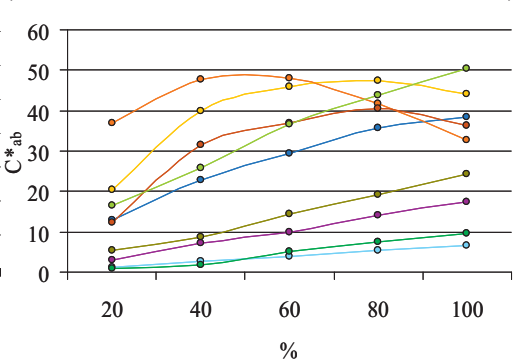

B.3.

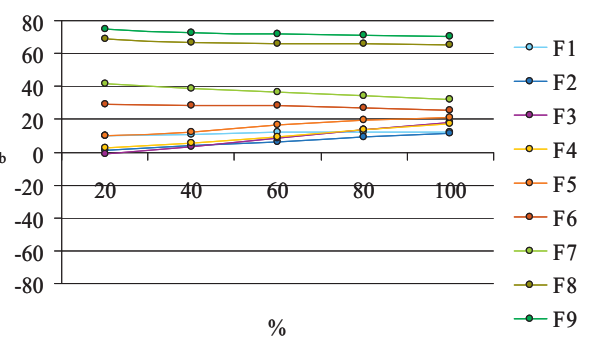

C.3.

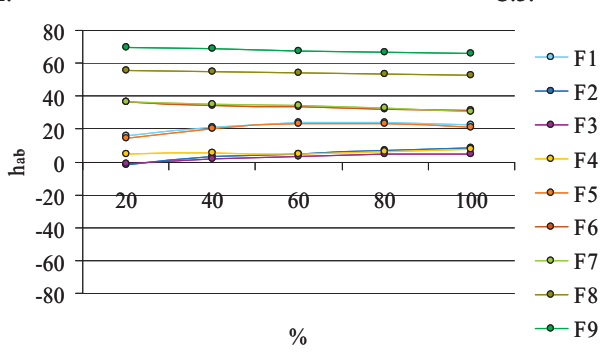

D.3.

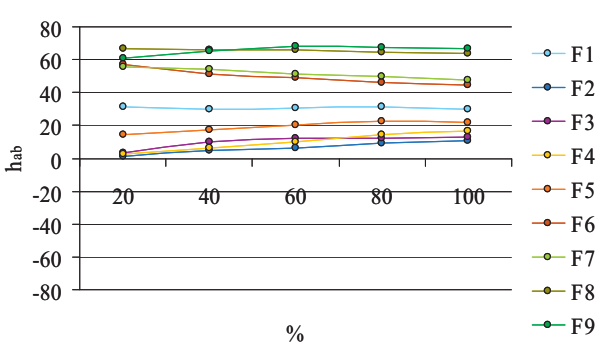

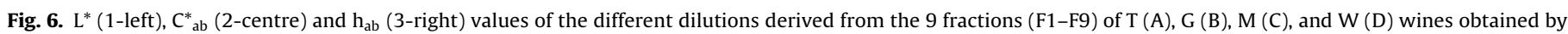
spectroradiometry. (For interpretation of the references to colour in this figure text, the reader is referred to the web version of the article.)

for each wine) to check for differences among the wines (Table 2). The results revealed that the $\mathrm{G}$ wine had significantly $(p<0.05)$ higher total pigment contents $\left(\sim 874.48 \mathrm{mg} \mathrm{L}^{-1}\right)$ than the $\mathrm{T}$ wine $\left(\sim 693.77 \mathrm{mg} \mathrm{L}^{-1}\right)$. Total pigment contents in the $\mathrm{M}$ and $\mathrm{W}$ wines were similar ( $\sim 746.03 \mathrm{mg} \mathrm{L}^{-1}$ and $\sim 749.76 \mathrm{mg} \mathrm{L}^{-1}$, respectively), and these values were significantly different from the total pigment contents in the $\mathrm{T}$ and $\mathrm{G}$ wines. These results are consistent with those obtained in previous studies carried out in wines [36].

\subsection{Effect of dilution on the colour of pigment fractions: application of different colorimetric techniques}

We performed colour measurements by applying the three colorimetric techniques (transmission spectrophotometry, diffuse reflectance spectrophotometry and spectroradiometry) described above on the different dilutions generated from the fractions obtained from the T, G, M and $\mathrm{W}$ wines.

Fig. 4 shows the values obtained using transmission spectrophotometry of the $\mathrm{L}^{*}, \mathrm{C}^{*}{ }_{\mathrm{ab}}$ and $\mathrm{h}_{\mathrm{ab}}$ colour parameters of the fractions of the $T, G, W$ and $M$ wines. As expected, with dilution lightness, $L^{*}$, increased in all the fractions of the T (Fig. 4 (A.1)), G (Fig. 4(B.1)), $\mathrm{M}$ (Fig. 4(C.1)) and W (Fig. 4(D.1)) wines, with the exception of fractions that without dilution (100\% of fraction) already showed values of lightness close to 100 , as F1, i.e., almost colourless fractions.

By contrast, the values of $\mathrm{C}^{*}{ }_{\mathrm{ab}}$ (Fig. 4(A.2-D.2)) decreased, this decrease being greater in the fractions with the higher pigment contents (F4-F6). Regarding the values of $h_{a b}$ (Fig. 4(A.3-D.3)), in general these were not seen to be affected by the dilution effect of the fractions, in agreement with the qualitative nature of this parameter. This shows that no modifications occurred in the anthocyanin equilibriums upon diluting under fixed $\mathrm{pH}$ conditions.

Regarding the colorimetric parameters obtained by diffuse reflectance (Fig. 5), it may be seen that, likewise, the dilution effect led to an increase in the values of $\mathrm{L}^{*}$ (Fig. 5(A.1-D.1)). Additionally, as with the spectrophotometer, the values of $h_{a b}$ (Fig. 5(A.3-D.3)) remained constant while the values of $C^{*}{ }_{a b}$ (Fig. 5(A.2-D.2)) mainly showed the opposite trend with dilution, in agreement with its role 

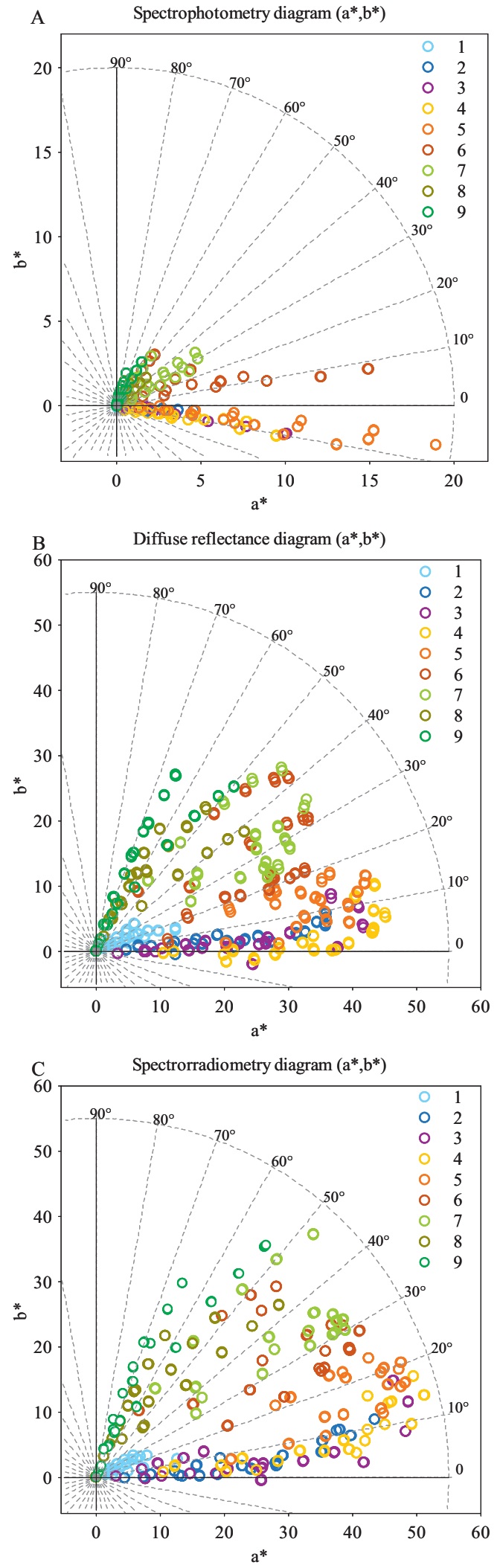

Fig. 7. Location of the wine fractions on the $\left(a^{*}, b^{*}\right)$-diagram. Spectrophotometry (A), diffuse reflectance (B), and spectroradiometry (C). (For interpretation of the references to colour in this figure text, the reader is referred to the web version of the article.) as a variable related to the chromatic intensity of vividness of the sample.

The variations in the values of the colour parameters were less marked with the measurements performed with diffuse reflectance (Fig. 5) than those observed when using transmittance (Fig. 4). However, with this latter technique it was possible to differentiate the fractions with high dilutions percentages better.

With respect to the measurements of colour using spectroradiometry, the results are shown in Fig. 5. The trend of the $L^{*}$ (Fig. 6(A.1-D.1)), $C^{*}$ ab (Fig. 6(A.2-D.2)) and $h_{a b}$ (Fig. 6(A.3-D.3)) colour parameters are similar to those observed with the other two techniques. However spectroradiometry allowed us to note the differences in the dilution effect on the fractions more clearly than diffuse reflectance (very similar) and transmittance.

The differences among colorimetric techniques with different measurement geometries (reflectance vs. transmittance) are well known in Tristimulus Colorimetry and correspond to the differences in visual appreciation, which depend on the observation geometry, such that in general they should not be interpreted as instrumental errors. Indeed, they are due to the different type of behaviour shown by light according to the angle of incidence on the sample, producing phenomena of reflection, transmission/absorption and refraction that clearly differ depending on the measurement in question.

Also, with a view to corroborating which colorimetric technique (transmission spectrophotometry, spectroradiometry and diffuse reflection spectrophotometry) allowed the samples to be differentiated better, regardless of the wine fractionated, we took as a reference the location on the diagram according to the $a^{*}$ and $b^{*}$ colour coordinates obtained. Thus, Fig. 7 shows the location of the samples on the $\left(a^{*}, b^{*}\right)$ plane. The samples were the dilutions obtained from each of the nine fractions from four wines. The transmission measurements (plot A) allowed us to distinguish only the dilutions obtained from fractions with higher contents in pigments (4-6) (Table 2). However, the distribution of the other fractions was better with the reflection measurements (plot B and plot C).

Diffuse reflectance spectrophotometry is a highly reproducible technology since it allows better control of the measuring conditions (environmental light or illumination/detection measuring geometry). It is a "contact" method in which the sample is directly attached to the analytical probe, blocking the measurement orifice, and so avoiding the influence of environmental light. Thus, the sample is illuminated only with the instrument's lamp, and hence this technique is more adequate for analytical objectives.

On the other hand, spectroradiometry reproduces the colour evaluation like the human eye does, existing certain distance between the measurement probe and the sample ("non-contact" method), so, the sample receives environmental light. Thus, this methodology better reproduces the differences in colour (such as the human eye would do) of fractions 4-6 and the rest of fractions.

\section{Conclusions}

The dilution effect led to an increase in $\mathrm{L}^{*}$, while the values of $\mathrm{C}^{*}{ }_{\mathrm{ab}}$ followed the opposite trend, in agreement with its role as a variable related to the chromatic intensity or vividness of the sample. The hue $h_{a b}$ did not seem to be affected by the dilution effect of the fractions, which is consistent with the qualitative nature of this parameter.

On the other hand, from a methodological point of view, the colorimetric differences between fractions were better distinguished by using spectroradiometry, so, it can be considered that this is the 
most adequate technique to assess and interpret or compare data obtained by sensory (visual) evaluation.

\section{Acknowledgements}

Thanks are due to Spanish CICYT (AGL2005-07245-C03) and Bodegas Roda S.A. for supplying the wine samples.

\section{References}

[1] J. Blouin, E. Peynaud, Enología Práctica Conocimiento y Elaboración del Vino, fourth ed., Mundi-Prensa Libros, S.A, Madrid, 2003.

[2] E. Peynaud, J. Blouin, El Gusto del Vino: el Gran Libro de la Degustación, second ed., Mundi-Prensa, Madrid, 2002.

[3] I. Esparza, C. Santamaría, J.M. Fernández, Anal. Chim. Acta 563 (2006) 331

[4] M.G. Jackson, C.F. Timberlake, P. Bridle, L. Vallis, J. Sci. Food Agric. 29 (1978) 715.

[5] T.C. Somers, Vitis 17 (1978) 161.

[6] R.B. Boulton, Am. J. Enol. Viticult. 52 (2001) 67

[7] R. Brouillard, S. Chassaing, A. Fougerousse, Phytochemistry 64 (2003) 1179-1186.

[8] E. Haslam, in: E. Haslam (Ed.), Practical Polyphenolics: From Structure to Molecular Recognition and Physiological Action, Press Syndicate of the University of Cambridge, Cambridge, 1998, pp. 262-297.

[9] B.S. Henry, in: G.A.F. Hendry, J.D. Houghton (Eds.), Natural Food Colorants, Blackie Academic \& Professional, London, 1996, pp. 40-79.

[10] R.L. Jackman, J.L. Smith, in: G.A.F. Hendry, J.D. Houghton (Eds.), Natural Food Colorants, second ed., Blackie Academic \& Professional, Chapman \& Hall, London, 1996, pp. 244-280.

[11] A.L. Waterhouse, V.F. Laurie, Am. J. Enol Viticult. 57 (2006) 306-313.

[12] G. Mazza, E. Miniati, in: G. Mazza, E. Miniati (Eds.), Anthocyanins in Fruit, Vegetables and Grains, CRC Press, Boca Raton, FL, 1993, pp. 1-199.

[13] E. Haslam, Phytochemistry 16 (1980) 1625.

[14] J.C. Rivas-Gonzalo, S. Bravo-Haro, C. Santos-Buelga, J. Agric. Food Chem. 43 (1995) 1444.
[15] M.T. Escribano-Bailón, O. Dangles, R. Brouillard, Phytochemistry 41 (1996) 1583.

[16] C. Santos-Buelga, E.M. Francia-Aricha, S. De Pascual Teresa, J.C. Rivas-Gonzalo, Eur. Food Res. Technol. 209 (1999) 411.

[17] M. Monagas, P.J. Martín-Álvarez, B. Bartolomé, C. Gómez-Cordovés, Eur. Food Res. Technol. 222 (2006) 702.

[18] M. Monagas, B. Bartolomé, in: M.V. Moreno-Arribas, M.C. Polo (Eds.), Wine Chemistry and Biochemistry, Springer, New York, 2009, pp. 439-462.

[19] Y. Glories, Connaiss. Vigne Vin 18 (1984) 195.

[20] P. Sudraud, Ann. Technol. Agric. 7 (1958) 203.

[21] A.I. Negueruela, J.F. Echávarri, M.M. Pérez, Am. J. Enol. Viticult. 46 (1995) 353.

[22] J.F. Gonnet, Food Chem. 63 (1998) 409.

[23] Commission International de l'Eclairage, Technical Report. Colorimetry. CIE 15:2004, third ed., Central Bureau, Viena, Austria, 2004.

[24] J. Bakker, C.F. Timberlake, J. Agric. Food Chem. 45 (1997) 35.

[25] F.J. Heredia, E.M. Francia-Aricha, J.C. Rivas-Gonzalo, I.M. Vicario, C. SantosBuelga, Food Chem. 63 (1998) 491.

[26] J.F. Gonnet, Food Chem. 66 (1999) 387.

[27] J.F. Gonnet, Food Chem. 75 (2001) 473.

[28] M.M. Giusti, L.E. Rodríguez-Saona, R.E. Wrolstad, J. Agric. Food Chem. 47 (1999) 4631.

[29] F.C. Stintzing, A.S. Stintzing, R. Carle, B. Frei, R.E. Wrolstad, J. Agric. Food Chem. 50 (2002) 6172.

[30] A. Terrab, M.L. González-Miret, F.J. Heredia, Eur. Food Res. Technol. 218 (2004) 488.

[31] A.J. Meléndez-Martínez, G. Britton, I.M. Vicario, F.J. Heredia, Food Chem. 101 (2007) 1145.

[32] OIV, Récuil des Méthodes Internationales d'Analyse des vins et des Moûts, Office International de la Vigne et du Vin, Paris, 2009.

[33] P. Ribereau-Gayon, E. Stonestreet, Bull. Soc. Chim. Fr. 9 (1965) 2649-2652.

[34] Y. Glories, Connaiss. Vigne Vin 18 (1984) 253-271.

[35] C. Alcalde-Eón, E. Boido, F. Carrau, E. Dellacassa, J.C. Rivas-Gonzalo, Am. J. Enol. Viticult. 57 (2006) 449.

[36] M. García-Marino, J.M. Hernández-Hierro, J.C. Rivas-Gonzalo, M.T. EscribanoBailón, Anal. Chim. Acta 660 (2010) 134.

[37] F.J. Heredia, C. Álvarez, M.L. González-Miret, A. Ramirez, CromaLab ${ }^{\circledR}$, Análisis de Color, Registro General de la Propiedad Intelectual SE-1052-04, Sevilla, Spain, 2004. 